\title{
Transmission wavefront shearing interferometry for photoelastic materials
}

\author{
Sharlotte L. B. Kramer, ${ }^{*}$ Guruswami Ravichandran, and Kaushik Bhattacharya \\ California Institute of Technology, Division of Engineering and Applied Science, \\ 1200 East California Boulevard, Pasadena, California 91125, USA \\ ${ }^{*}$ Corresponding author: sharlott@ caltech.edu
}

Received 6 November 2008; revised 16 March 2009; accepted 19 March 2009; posted 20 March 2009 (Doc. ID 103187); published 23 April 2009

\begin{abstract}
A general analysis and experimental validation of transmission wavefront shearing interferometry for photoelastic materials are presented. These interferometers applied to optically isotropic materials produce a single interference pattern related to one phase term, but when applied to photoelastic materials, they produce the sum of two different interference patterns with phase terms that are the sum and difference, respectively, of two stress-related phase terms. The two stress-related phase terms may be separated using phase shifting and polarization optics. These concepts are experimentally demonstrated using coherent gradient sensing in full field for a compressed polycarbonate plate with a V-shaped notch with good agreement with theoretical data. The analysis may be applied to any wavefront shearing interferometer by modifying parameters describing the wavefront shearing distance. (c) 2009 Optical Society of America

OCIS codes: $\quad 120.4880,120.7000,160.4760,260.1440,260.5430$.
\end{abstract}

\section{Introduction}

Wavefront shearing interferometry is a wellestablished optical technique for measuring many optical, material, and mechanical properties such as wavefront slope characterization [1], surface deformation [2], and even fracture of materials [3-6]. Shearing interferometry essentially is the interference of a coherent wavefront with a copy of itself "sheared" or translated by a distance $d_{\text {shear }}$; this technique is self-referencing and hence is insensitive to rigid body motion [2-5]. The general analysis of the interference pattern for standard wavefront shearing interferometers depends only on the wavefront characteristics and the distance $d_{\text {shear }}$. Once the parameters for producing the sheared wavefront and interfering the two wavefronts are characterized for a particular shearing method, then the analysis may be detailed for that method. With several methods to produce the wavefront shearing, the choice of shearing interferometer depends on the requirements of

0003-6935/09/132450-11\$15.00/0

(C) 2009 Optical Society of America the application such as measurement sensitivity or compactness.

An important consideration for the analysis is how the wavefront is formed. For techniques that involve transmission through a material of interest, the shape and optical properties of the material are considered (e.g., a spherical wavefront emanating from an optically isotropic plano-convex lens.) In the case of a deformed material that is originally planar, thickness and refractive index variations in the material result in optical path differences that may be related to stresses. A general analysis of the optical path difference in this case was previously completed for the method of caustics [7-9]. Though not a wavefront shearing interferometry technique, the method of caustics, which has been used for large stress gradient applications, does consider optical path differences due to a deformed sample, resulting in a shadow spot in the far field. The method of caustics gives only a point measurement, which motivated the development of coherent gradient sensing (CGS), capable of measuring full-field stress or displacement gradients when used in transmission or in reflection, respectively [3,4]. CGS is a wavefront 
lateral shearing interferometer that achieves shearing by a pair of amplitude gratings; sensitivity adjustment is achievable through choice of grating line density, separation between the gratings, and light wavelength. Previously, CGS in transmission has been used only for optically isotropic materials $[3,4,6]$. CGS in reflection has been used for opaque isotropic materials $[3,4]$, for materials with reflective coatings $[3,4,10]$, and for composite materials $[\underline{5}, \underline{11}]$. No previous studies have considered CGS in transmission for optically anisotropic materials.

Taking inspiration from the method of caustics applied to photoelastic materials, this paper presents what is to our knowledge the first general analysis of an initially planar wavefront transmitted through a photoelastic material, in terms of electric field and optical path difference, for a general wavefront shearing interferometer; the analysis is then specifically applied to CGS. The analysis may easily be modified for any wavefront shearing interferometer by changing the experimental parameters related to the distance $d_{\text {shear }}$.

This study demonstrates that the resultant interference pattern is no longer a simple function of a single phase term related to the sum of principal stresses, denoted $\varphi_{\text {sum }}$, as in the case of optically isotropic materials. Due to the optical anisotropy from the stress birefringence, the interference patterns from the $x$ and $y$ coordinates of the electric field, $E_{x}$ and $E_{y}$, respectively, are no longer equivalent. Considering the interference patterns along the orthogonal principal axes of the photoelastic specimen, denoted $I_{1}^{\text {image }}$ and $I_{2}^{\text {image }}$, the phase terms of these distinct interference patterns, $\varphi_{1}$ and $\varphi_{2}$, are $\varphi_{\text {sum }}+\varphi_{\text {diff }}$ and $\varphi_{\text {sum }}-\varphi_{\text {diff }}$, respectively, where $\varphi_{\text {diff }}$ is related to the difference of principal stresses. Thus, $\varphi_{\text {diff }}$ obscures the desired phase information, $\varphi_{\text {sum }}$, due to the optical anisotropy of the material. $\varphi_{\text {diff }}$ is zero for an optically isotropic material and therefore is not an issue for isotropic materials. For a general incident electric field, wavefront shearing interferometry for photoelastic materials results in an image that is the superposition of $I_{1}^{\text {image }}$ and $I_{2}^{\text {image }}$, which is too complicated to analyze by itself. The desired phase $\varphi_{\text {sum }}$ may be recovered by using phase shifting and polarization optics. These concepts are demonstrated using CGS for a compressed polycarbonate thin plate with a V-shaped side notch with good agreement between experimental and theoretical data.

\section{Experimental Method and Analysis of Full-Field Phase Data}

\section{A. Experimental Method}

The CGS method starts with an incident plane wave of a collimated laser beam that transmits through a transparent sample or that reflects off an opaque sample. The working principle of CGS to laterally shear an incident wavefront, shown in Fig. 1 for horizontal shear, is the same for both transmission and

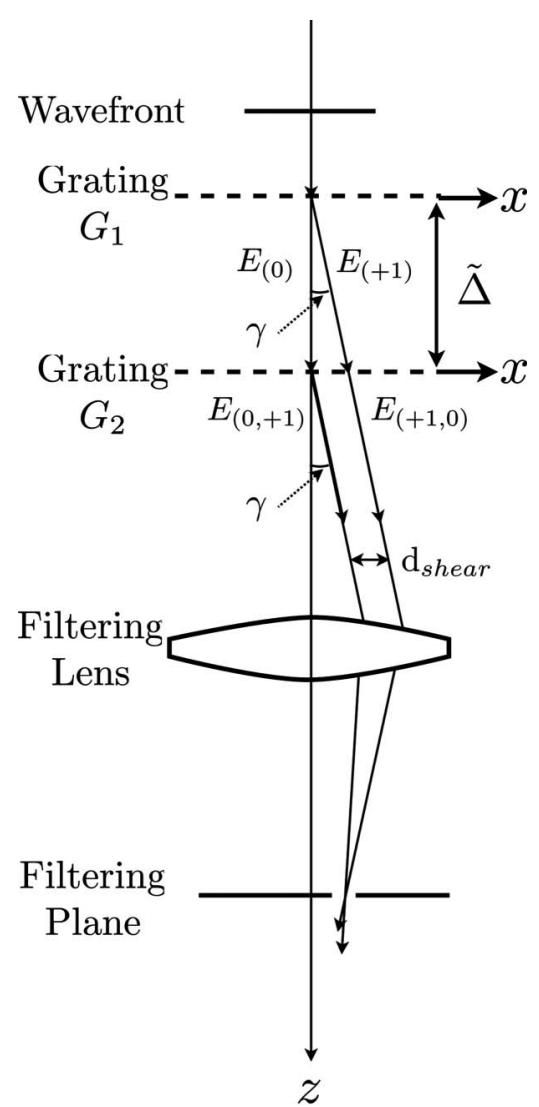

Fig. 1. Working principle for horizontal shearing transmission coherent gradient sensing.

reflection. Tippur et al. [3,4] give a full description of the CGS working principle. The main concept of CGS is that the $d_{\text {shear }}$ of the interfered wavefronts is due to diffraction through a pair of Ronchi gratings, $G_{1}$ and $G_{2}$, each with pitch $p$, separated by distance $\Delta$ such that the desired wavefronts $\boldsymbol{E}_{(\mathbf{0}, \pm \mathbf{1})}$ and $\boldsymbol{E}_{( \pm \mathbf{1 , 0})}$ are separated by a lateral shearing distance $d_{\text {shear }}=\gamma \Delta$ in the $x-z$ or $y-z$ plane and propagate at the same angle $\gamma$ relative to the $z$ axis upon leaving grating $G_{2}$. The diffracted waves transmit through a filtering lens, which separates the corresponding diffraction orders into horizontal diffraction spots at the focal plane of the filtering lens. An aperture at this focal plane selects either the +1 or -1 diffraction order, meaning only the wavefronts $\boldsymbol{E}_{(\mathbf{0}, \pm \mathbf{1})}$ and $\boldsymbol{E}_{( \pm \mathbf{1 . 0})}$ propagate to the image plane. In Subsection 2.B, analysis of the first-order diffraction shows how the interference pattern may be related to the first $x$ and $y$ derivatives of principal stresses based on assumption of a small $d_{\text {shear }}$.

\section{B. Analysis}

\section{Electric Field Description of the Transmitted Wavefront}

Assuming a coherent plane wave of monochromatic light propagating along the $z$ axis, the electric field of the wavefront at $z=z_{o}$ is given by 


$$
\begin{aligned}
& E^{\text {in }}(x, y, t)=E_{x}(x, y, t) \hat{\imath}+E_{y}(x, y, t) \bigotimes, \\
& E_{x}(x, y, t)=A_{x} \exp \left[j\left(k z_{o}-\omega t+\phi_{x}\right)\right], \\
& E_{y}(x, y, t)=A_{y} \exp \left[j\left(k z_{o}-\omega t+\phi_{y}\right)\right],
\end{aligned}
$$

where $E_{x}$ and $E_{y}$ are the amplitudes, $A_{x}$ and $A_{y}$ are constants, $\lambda$ is the wavelength, $k=2 \pi / \lambda$ is the wavenumber, $\omega$ is the angular frequency, and $\phi_{x}$ and $\phi_{y}$ are arbitrary constant phase terms. If the plane wave propagates through a transparent material with refractive index $n_{o}$ and nominal thickness $h$, then the resulting electric field magnitudes of this perturbed wavefront in the $x$ and $y$ directions after the sample material at $z$ are

$$
\begin{aligned}
E_{x}^{\text {sample }}(x, y, t)= & A_{x} \exp \left[j \left(k z-\omega t+\phi_{x}\right.\right. \\
& \left.\left.+k\left(n_{o}-1\right) h+k \Delta S_{x}(x, y)\right)\right], \\
E_{y}^{\text {sample }}(x, y, t)= & A_{y} \exp \left[j \left(k z-\omega t+\phi_{y}\right.\right. \\
& \left.\left.+k\left(n_{o}-1\right) h+k \Delta S_{y}(x, y)\right)\right],
\end{aligned}
$$

where $\Delta S_{x}(x, y)$ and $\Delta S_{y}(x, y)$ are the optical path differences at each point $(x, y)$ along the $x$ and $y$ directions, as further described in Subsection 2.B.2.

\section{Photoelastic Effect in Transparent Materials}

In general, a plane wave transmitted through a material experiences some change in optical path length due to both variation in refractive index, $\Delta n(x, y)$, and variation in thickness, $\Delta h(x, y)$, in the transmitting media. Along a given axis $a$, the optical path difference is expressed as

$$
\Delta S_{a}(x, y)=h \Delta n_{a}(x, y)+\left(n_{o}-1\right) \Delta h(x, y) .
$$

A full explanation of the optical path difference may be found in [7]. These variations from an initially uniform material can be related to stresses in the material. First, a transparent material that experiences stress-induced birefringence, also known as the photoelastic effect, has variations in refractive index along the three principal optical axes such that

$$
\begin{aligned}
& \Delta n_{1}=n_{1}-n_{o}=A \sigma_{1}+B\left(\sigma_{2}+\sigma_{3}\right), \\
& \Delta n_{2}=n_{2}-n_{o}=A \sigma_{2}+B\left(\sigma_{1}+\sigma_{3}\right), \\
& \Delta n_{3}=n_{3}-n_{o}=A \sigma_{3}+B\left(\sigma_{1}+\sigma_{2}\right),
\end{aligned}
$$

where $\sigma_{i}, i=\{1,2,3\}$, are the principal stresses and $A$ and $B$ are the two absolute photoelastic constants of the transparent material. These equations are known as the Neumann-Maxwell stress optic law [12-14]. In this analysis, the $\hat{p_{3}}$ principal direction is assumed to be along the $z$ axis. Second, the thickness change in a linear elastic material is related to the principal stresses by Hooke's law:

$$
\Delta h=\left[\frac{\sigma_{3}}{E}-\frac{\nu}{E}\left(\sigma_{1}+\sigma_{2}\right)\right] h,
$$

where $E$ is Young's modulus, $\nu$ is the Poisson ratio, $\sigma_{3}=0$ for plane stress, and $\Delta h=0$ for plane strain.

Substituting Eqs. (4a), (4b), and (ㅁ) into Eq. (3) results in the following two equations for optical path length difference along the $\hat{p_{1}}$ and $\hat{p_{2}}$ principal directions in terms of the sum and difference of principal stresses:

$$
\begin{aligned}
& \Delta S_{1}(x, y)=C h\left[\left(\sigma_{1}+\sigma_{2}\right)+g\left(\sigma_{1}-\sigma_{2}\right)\right], \\
& \Delta S_{2}(x, y)=C h\left[\left(\sigma_{1}+\sigma_{2}\right)-g\left(\sigma_{1}-\sigma_{2}\right)\right],
\end{aligned}
$$

such that $C=[(A+B) / 2]-\left[(\nu / E)\left(n_{o}-1\right)\right]$ and $g=$ $(A-B) /\left[A+B-2 \nu\left(n_{o}-1\right) / E\right]$ for plane stress and $C=[(A+B) / 2]+\nu B$ and $g=(A-B) /(A+B+2 \nu B)$ for plane strain. For optically isotropic (nonbirefringent) materials, $A=B$, resulting in $g=0$; thus, in this case, $\Delta S_{1}(x, y)=\Delta S_{2}(x, y)=\Delta S(x, y)$. For optically anistropic (birefringent) materials, $A \neq B$; thus, $\Delta S_{1}(x, y) \neq \Delta S_{2}(x, y)$ in general.

\section{Electric Field of the Transmitted Wavefront}

The incident wavefront given in Eq. (1) may be written in the orthogonal principal coordinate system at each point $(x, y)$, such that

$$
\begin{gathered}
\boldsymbol{E}_{p}^{\mathrm{in}}(x, y, t)=E_{1}(x, y, t) \hat{p_{1}}+E_{2}(x, y, t) \hat{p_{2}}, \\
E_{1}(x, y, t)=E_{x}(x, y, t) \cos (\alpha)+E_{y}(x, y, t) \sin (\alpha), \\
E_{2}(x, y, t)=-E_{x}(x, y, t) \sin (\alpha)+E_{y}(x, y, t) \cos (\alpha), \\
\hat{p_{1}}=\cos (\alpha) \hat{i}+\sin (\alpha) \bigotimes, \\
\hat{p_{2}}=-\sin (\alpha) \hat{i}+\cos (\alpha) \bigotimes,
\end{gathered}
$$

where $\alpha$ is the angle between the Cartesian and the principal coordinate systems. The effect of transmission through a birefringent plate is the gain of a phase of $k \Delta S_{1,2}$ along the principal directions, resulting in a transmitted wavefront in the principal coordinate system of

$$
\begin{aligned}
\boldsymbol{E}_{p}^{\text {sample }}(x, y, t)= & E_{1}(x, y, t) \exp \left[j k \Delta S_{1}(x, y)\right] \hat{p_{1}} \\
& +E_{2}(x, y, t) \exp \left[j k \Delta S_{2}(x, y)\right] \hat{p_{2}}
\end{aligned}
$$




\section{Analysis of Interference Pattern}

As described in Subsection 2.A, the interference of wavefronts $\boldsymbol{E}_{(\mathbf{0}, \pm \mathbf{1})}$ and $\boldsymbol{E}_{( \pm \mathbf{1}, \mathbf{0})}$ is the interference of two identical wavefronts $\boldsymbol{E}^{ \pm 1}$ that are separated by distance $d_{\text {shear }}$, as written in Eqs. (9) for the lateral shearing of the electric field in the $x$ direction with the electric field in the principal coordinate system:

$$
\begin{aligned}
E_{p}^{\text {image }}(x, y)= & E_{1}^{\text {image }}(x, y) \hat{p_{1}}+E_{2}^{\text {image }}(x, y) \hat{p_{2}}, \\
E_{1}^{\text {image }}(x, y)= & E_{1}^{ \pm 1}(x, y)+E_{1}^{ \pm 1}\left(x+d_{\text {shear }}, y\right), \\
E_{2}^{\text {image }}(x, y)= & E_{2}^{ \pm 1}(x, y)+E_{2}^{ \pm 1}\left(x+d_{\text {shear }}, y\right), \\
E_{1}^{ \pm 1}(x, y)= & A_{x}^{ \pm 1} \cos (\alpha) \exp \left[j \left(k z-\omega t+\phi_{x}\right.\right. \\
& \left.\left.+k \Delta S_{1}(x, y)\right)\right] \\
& +A_{y}^{ \pm 1} \sin (\alpha) \exp \left[j \left(k z-\omega t+\phi_{y}\right.\right. \\
& \left.\left.+k \Delta S_{1}(x, y)\right)\right], \\
& \\
E_{2}^{ \pm 1}(x, y)= & A_{x}^{ \pm 1} \sin (\alpha) \exp \left[j \left(k z-\omega t+\phi_{x}\right.\right. \\
& \left.\left.+k \Delta S_{2}(x, y)\right)\right] \\
& +A_{y}^{ \pm 1} \cos (\alpha) \exp \left[j \left(k z-\omega t+\phi_{y}\right.\right. \\
& \left.\left.+k \Delta S_{2}(x, y)\right)\right],
\end{aligned}
$$

where constants $A_{x}^{ \pm 1}<A_{x}$ and $A_{y}^{ \pm 1}<A_{y}$ due to diffraction. The resulting irradiance (intensity) of the interfered wavefronts, $I^{\text {image }}$, in Eqs. (10), is the superposition of the irradiance of the $E_{1}$ component, $I_{1}^{\text {image }}$, and the irradiance of the $E_{2}$ component, $I_{2}^{\text {image }}$, since the principal directions are orthogonal:

$$
\begin{aligned}
I^{\text {image }} & =\left\langle E_{1}^{\text {image }} E_{1}^{\text {image* }}\right\rangle_{t}+\left\langle E_{2}^{\text {image }} E_{2}^{\text {image* }}\right\rangle_{t} \\
& =I_{1}^{\text {image }}+I_{2}^{\text {image }}
\end{aligned}
$$

Therefore, the resultant image is the following:

$$
\begin{aligned}
I^{\text {image }}= & 2\left(A_{x}^{ \pm 1}\right)^{2}+2\left(A_{y}^{ \pm 1}\right)^{2} \\
& +\left\{2\left(A_{x}^{ \pm 1}\right)^{2} \cos ^{2}(\alpha)+2\left(A_{y}^{ \pm 1}\right)^{2} \sin ^{2}(\alpha)\right. \\
& \left.+2 A_{x}^{ \pm 1} A_{y}^{ \pm 1} \cos (\alpha) \sin (\alpha) \cos \left(\phi_{x}-\phi_{y}\right)\right\} \\
& \times \cos \left[k\left(\Delta S_{1}(x, y)-\Delta S_{1}\left(x+d_{\text {shear }}, y\right)\right)\right] \\
& +2\left(A_{x}^{ \pm 1}\right)^{2} \sin ^{2}(\alpha)+2\left(A_{y}^{ \pm 1}\right)^{2} \cos ^{2}(\alpha) \\
& \left.-2 A_{x}^{ \pm 1} A_{y}^{ \pm 1} \cos (\alpha) \sin (\alpha) \cos \left(\phi_{x}-\phi_{y}\right)\right\} \\
& \times \cos \left[k\left(\Delta S_{2}(x, y)-\Delta S_{2}\left(x+d_{\text {shear }}, y\right)\right)\right] .
\end{aligned}
$$

The shearing distance is usually small compared to the field of view of the image $(\mathrm{L} \times \mathrm{W})$, so the phase terms of $I_{1}^{\mathrm{image}}$ and $I_{2}^{\mathrm{image}}$, denoted $\varphi_{1,2}(x, y)$, can be related to the derivatives of $\Delta S_{1,2}$. For $\left(d_{\text {shear }} /\{L, W\}\right) \ll 1$,

$$
\begin{aligned}
\varphi_{1,2} & =k\left(\Delta S_{1,2}(x, y)-\Delta S_{1,2}(x+\Delta x, y)\right) \\
& \approx k d_{\text {shear }} \frac{\partial \Delta S_{1,2}(x, y)}{\partial x} .
\end{aligned}
$$

Substituting $\Delta S_{1,2}$ from Eqs. (6a) and (6b) into Eq. (12) connects the phase terms of the interference patterns to stresses:

$$
\varphi_{1,2}=k d_{\text {shear }} C h\left[\frac{\partial\left(\sigma_{1}+\sigma_{2}\right)}{\partial x} \pm g \frac{\partial\left(\sigma_{1}-\sigma_{2}\right)}{\partial x}\right]
$$

The equation for the image may be written in terms of two phases, one related to $\sigma_{1}+\sigma_{2}$ and the other related to $\sigma_{1}-\sigma_{2}$ as follows:

$I^{\text {image }}=I_{o}+I_{1 o} \cos \left[\varphi_{\text {sum }}+\varphi_{\text {diff }}\right]+I_{2 o} \cos \left[\varphi_{\text {sum }}-\varphi_{\text {diff }}\right]$,

$$
I_{o}=2\left(A_{x}^{ \pm 1}\right)^{2}+2\left(A_{y}^{ \pm 1}\right)^{2}
$$

$$
\begin{aligned}
I_{1}^{\text {image }}= & 2\left(A_{x}^{ \pm 1}\right)^{2} \cos ^{2}(\alpha)+2\left(A_{y}^{ \pm 1}\right)^{2} \sin ^{2}(\alpha)+4 A_{x}^{ \pm 1} A_{y}^{ \pm 1} \cos (\alpha) \sin (\alpha) \cos \left(\varphi_{x}-\varphi_{y}\right) \\
& +\left\{2\left(A_{x}^{ \pm 1}\right)^{2} \cos ^{2}(\alpha)+2\left(A_{y}^{ \pm 1}\right)^{2} \sin ^{2}(\alpha)\right\} \cos \left[k \Delta S_{1}(x, y)-k \Delta S_{1}\left(x+d_{\text {shear }}, y\right)\right] \\
& +\left\{2 A _ { x } ^ { \pm 1 } A _ { y } ^ { \pm 1 } \operatorname { c o s } ( \alpha ) \operatorname { s i n } ( \alpha ) \left\{\cos \left[\phi_{x}-\phi_{y}+k \Delta S_{1}(x, y)-k \Delta S_{1}\left(x+d_{\text {shear }}, y\right)\right]\right.\right. \\
& \left.+\cos \left[\phi_{y}-\phi_{x}+k \Delta S_{1}(x, y)-k \Delta S_{1}\left(x+d_{\text {shear }}, y\right)\right]\right\} \\
I_{2}{ }^{\text {image }}= & 2\left(A_{x}^{ \pm 1}\right)^{2} \sin ^{2}(\alpha)+2\left(A_{y}^{ \pm 1}\right)^{2} \cos ^{2}(\alpha)-4 A_{x}^{ \pm 1} A_{y}^{ \pm 1} \cos (\alpha) \sin (\alpha) \cos \left(\varphi_{x}-\varphi_{y}\right) \\
& +\left\{2\left(A_{x}^{ \pm 1}\right)^{2} \sin ^{2}(\alpha)+2\left(A_{y}^{ \pm 1}\right)^{2} \cos ^{2}(\alpha)\right\} \cos \left[k \left(\Delta S_{2}(x, y)-k\left(\Delta S_{2}\left(x+d_{\text {shear }}, y\right)\right]\right.\right. \\
& +2 A_{x}^{ \pm 1} A_{y}^{ \pm 1} \cos (\alpha) \sin (\alpha)\left\{\cos \left[\phi_{x}-\phi_{y}+k \Delta S_{2}(x, y)-k \Delta S_{2}\left(x+d_{\text {shear }}, y\right)\right]\right. \\
& \left.+\cos \left[\phi_{y}-\phi_{x}+k \Delta S_{2}(x, y)-k \Delta S_{2}\left(x+d_{\text {shear }}, y\right)\right]\right\}
\end{aligned}
$$




$$
\begin{gathered}
I_{1 o}=2\left(A_{x}^{ \pm 1}\right)^{2} \cos ^{2}(\alpha)+2\left(A_{y}^{ \pm 1}\right)^{2} \sin ^{2}(\alpha) \\
+2 A_{x}^{ \pm 1} A_{y}^{ \pm 1} \cos (\alpha) \sin (\alpha) \cos \left(\phi_{x}-\phi_{y}\right), \\
I_{2 o}=2\left(A_{x}^{ \pm 1}\right)^{2} \sin ^{2}(\alpha)+2\left(A_{y}^{ \pm 1}\right)^{2} \cos ^{2}(\alpha) \\
-2 A_{x}^{ \pm 1} A_{y}^{ \pm 1} \cos (\alpha) \sin (\alpha) \cos \left(\phi_{x}-\phi_{y}\right), \\
\varphi_{\text {sum }}=k d_{\text {shear }} \operatorname{Ch} \frac{\partial\left(\sigma_{1}+\sigma_{2}\right)}{\partial x}, \\
\varphi_{\text {diff }}=k d_{\text {shear }} \operatorname{Chg} \frac{\partial\left(\sigma_{1}-\sigma_{2}\right)}{\partial x} .
\end{gathered}
$$

Since the intensity contains a sum of two sinusoids with the same frequency $k$, then Eq. (14a) may be written as a single interference pattern with a phase that is the sum of $\varphi_{\text {sum }}$ and a compound phase $\varphi_{c}$ :

$$
\begin{gathered}
I^{\text {image }}=I_{o}+I_{c} \cos \left[\varphi_{\mathrm{sum}}+\varphi_{c}\right] \\
I_{c}=\sqrt{I_{1 o}^{2}+I_{2 o}^{2}+2 I_{1 o} I_{2 o} \cos \left(2 \varphi_{\text {diff }}\right)}, \\
\varphi_{c}=\arctan \left[\frac{\left(I_{1 o}-I_{2 o}\right) \sin \left(\varphi_{\text {diff }}\right)}{\left(I_{1 o}+I_{2 o}\right) \cos \left(\varphi_{\text {diff }}\right)}\right]
\end{gathered}
$$

A similar result for the $y$ direction shearing may be obtained from the previous analysis, except the derivatives are with respect to $y$ instead of $x$.

For the specific case of CGS, $d_{\text {shear }}$ is $\tilde{\Delta} \lambda / p$, with $k=2 \pi / \lambda$, such that Eqs. (14e) and (14f) become the following:

$$
\begin{gathered}
\varphi_{\text {sum }}=\frac{2 \pi \tilde{\Delta} C h}{p} \frac{\partial\left(\sigma_{1}+\sigma_{2}\right)}{\partial x}, \\
\varphi_{\text {diff }}=\frac{2 \pi \tilde{\Delta} C h g}{p} \frac{\partial\left(\sigma_{1}-\sigma_{2}\right)}{\partial x} .
\end{gathered}
$$

For linearly elastic, optically isotropic materials with $g=0$, then $\varphi_{\text {diff }}=0$, which leads to the classic result for image irradiance, $I^{\text {isotropic }}=I_{o}\{1+$ $\left.\cos \left[\varphi_{\text {sum }}\right]\right\}$, where the phase term of the interference pattern is related only to the derivative of the sum of principal stresses [4]. As shown above, unlike optically isotropic materials, photoelastic materials produce complicated interference patterns that are difficult to interpret. Fortunately, phase shifting methods in conjunction with incident polarized light allow for the recovery of $\varphi_{\text {sum }}$, and thus the $x$ or $y$ derivative of $\sigma_{1}+\sigma_{2}$, in full field.

\section{Phase Separation and Interpretation}

\section{Four-Step Phase Shifting}

The phase shifting interferometry technique used for CGS in this study is a four-step technique with $\pi / 2$ phase steps, induced by a lateral shift of $p / 4$ in one Ronchi grating in the direction of the dominant lateral shearing, resulting in four phase shifted interference patterns. For an optically isotropic material, the resultant intensities, which are functions of a single phase term $\varphi$, are $I_{1}=$ $I_{o}(1+\cos (\varphi)), I_{2}=I_{o}(1+\cos (\varphi+\pi / 2)), I_{3}=I_{o}(1+$ $\cos (\varphi+\pi))$, and $I_{4}=I_{o}(1-+\cos (\varphi+3 \pi / 2))$. The original phase map, $\varphi$, is related to these intensities by

$$
\varphi=\arctan \left[\frac{I_{4}-I_{2}}{I_{1}-I_{3}}\right]=\arctan \left[\frac{\sin (\varphi)}{\cos (\varphi)}\right] .
$$

This equation yields a "wrapped" phase map with discontinuities of height $h_{d}=2 \pi$ since the range of an $\arctan ()$ formula is $2 \pi$ when the signs of the numerator and denominator are known. The full range of $\varphi$ is determined by unwrapping the phase term from the $\arctan ()$ formula, as described in Section 3.

For optically anisotropic materials for a general initial electric field, from Eq. (15), the four phase shifted images are

$$
\begin{gathered}
I_{1}=I_{o}+I_{c} \cos \left[\varphi_{\mathrm{sum}}+\varphi_{c}\right], \\
I_{2}=I_{o}+I_{c} \cos \left[\varphi_{\mathrm{sum}}+\varphi_{c}+\frac{\pi}{2}\right], \\
I_{3}=I_{o}+I_{c} \cos \left[\varphi_{\mathrm{sum}}+\varphi_{c}+\pi\right], \\
I_{4}=I_{o}+I_{c} \cos \left[\varphi_{\mathrm{sum}}+\varphi_{c}+\frac{3 \pi}{2}\right] .
\end{gathered}
$$

The phase map of $\varphi_{\text {sum }}+\varphi_{c}$ may be recovered using the typical $\arctan ()$ formula similar to Eq. (17) such that

$$
\begin{aligned}
\varphi_{\text {sum }}+\varphi_{c} & =\arctan \left[\frac{I_{4}-I_{2}}{I_{1}-I_{3}}\right] \\
& =\arctan \left[\frac{I_{c} \sin \left(\varphi_{\text {sum }}+\varphi_{c}\right)}{I_{c} \cos \left(\varphi_{\text {sum }}+\varphi_{c}\right)}\right],
\end{aligned}
$$

but Eq. (19) is indeterminate when $I_{c}=0$, so this equation is true only for $I_{c} \neq 0$. Specifically polarized input electric fields allow for separation of $\varphi_{\text {sum }}$ from $\varphi_{c}$, as discussed below.

\section{Two Methods for Determination of the First Derivative of $\sigma_{1}+\sigma_{2}$}

The first method to recover $\varphi_{\text {sum }}$ involves capturing images from a pure $E_{x} \hat{\imath}$ input electric field and from a 
pure $E_{y} \bigotimes$ input electric field. From Eq. (15), for $A_{x}=$ $A_{o}$ and $A_{y}=0$, and thus $A_{x}^{ \pm 1}=A_{o}^{ \pm 1}$ and $\overline{A_{y}^{ \pm 1}}=0$, the image is

$$
\begin{gathered}
I^{E x}=I_{o}^{E x}+I_{c}^{E x} \cos \left[\varphi^{E x}\right], \\
\varphi^{E x}=\varphi_{\text {sum }}+\varphi_{\alpha d}, \\
I_{o}^{E x}=2\left(A_{o}^{ \pm 1}\right)^{2}, \\
I_{c}^{E x}=I_{o}^{E x} \sqrt{1-\sin ^{2}(2 \alpha) \sin ^{2}\left(\phi_{\text {diff }}\right)}, \\
\varphi_{\alpha d}=\arctan \left[\cos (2 \alpha) \tan \left(\phi_{\text {diff }}\right)\right],
\end{gathered}
$$

where $\varphi_{\alpha d}$ is a compound phase related to $\alpha$ and $\varphi_{\text {diff }}$. Similarly, for $A_{x}=0$ and $A_{y}=A_{o}$, and thus $A_{x}^{ \pm 1}=0$ and $A_{y}^{ \pm 1}=A_{o}^{ \pm 1}$ from Eq. (15), the image is

$$
\begin{gathered}
I^{E y}=I_{o}^{E y}+I_{c}^{E y} \cos \left[\varphi^{E y}\right], \\
\varphi^{E y}=\varphi_{\mathrm{sum}}-\varphi_{\alpha d}, \\
I_{o}^{E y}=2\left(A_{o}^{ \pm 1}\right)^{2}, \\
I_{c}^{E y}=I_{o}^{E y} \sqrt{1-\sin ^{2}(2 \alpha) \sin ^{2}\left(\phi_{\text {diff }}\right)} .
\end{gathered}
$$

If phase shifted images for these two configurations are taken for the same field of view for the same deformation state in the sample, then the $\varphi^{E x}$ and $\varphi^{E y}$ fields are calculated by Eq. (19). For both of these fields, Eq. (19) does not hold for $\sqrt{1-\sin ^{2}(2 \alpha) \sin ^{2}\left(\phi_{\text {diff }}\right)}=0$, but this is likely true for only a few points in the field of view. Since $I_{c}^{E x}$ and $I_{c}^{E y}$ are always nonnegative, then Eq. (19) can express the absolute sign of the numerator and denominator separately for each configuration, and the height discontinuity of the wrapped phases is $h_{d}=2 \pi$, as explained in Subsection 2.C.1. After unwrapping these fields, $\varphi_{\text {sum }}$ may be separated from the other phase, meaning $\varphi_{\text {sum }}=\left(\varphi^{E x}+\varphi^{E y}\right) / 2 . \quad$ Additionally, $\quad \varphi_{\alpha d}=\left(\varphi^{E x_{-}}\right.$ $\left.\varphi^{E y}\right) / 2=\arctan \left[\cos (2 \alpha) \tan \left(\phi_{\text {diff }}\right)\right]$. Subsection 2.C.3 describes possible configurations of polarization optics to achieve this case.

Another possible method for determining $\varphi_{\text {sum }}$ requires only one set of phase shifted images. If the input electric field is circularly polarized such that $A_{x}=A_{y}=A_{o} / \sqrt{2}, \phi_{x}=\phi_{y} \pm \pi / 2$, and consequently $A_{x}^{ \pm 1}=A_{y}^{ \pm 1}=A_{o}^{ \pm 1} / \sqrt{2}$ using polarization optics, then the image given in Eq. (15) may be simplified to

$$
I^{\mathrm{circ}}=I_{o}^{\mathrm{circ}}+I_{c}^{\mathrm{circ}} \cos \left[\varphi_{\mathrm{sum}}\right],
$$

$$
I_{o}^{\text {circ }}=2\left(A_{o}^{ \pm 1}\right)^{2}
$$

$$
I_{c}^{\text {circ }}=I_{o}^{\text {circ }} \cos \left[\varphi_{\text {diff }}\right] .
$$

If phase shifted images for this configuration are analyzed using Eq. (19), then $\varphi_{\text {sum }}$ is determined by

$$
\begin{aligned}
\varphi_{\text {sum }} & =\arctan \left[\frac{I_{4}-I_{2}}{I_{1}-I_{3}}\right] \\
& =\arctan \left[\frac{\sin \left(\varphi_{\text {sum }}\right) \cos \left(\varphi_{\text {diff }}\right)}{\cos \left(\varphi_{\text {sum }}\right) \cos \left(\varphi_{\text {diff }}\right)}\right] .
\end{aligned}
$$

This equation is only true for $(x, y)$ coordinates where $\cos \left(\varphi_{\text {diff }}\right) \neq 0$, since the argument of the $\arctan ()$ is indeterminate where $\cos \left(\varphi_{\text {diff }}\right)=0$. Since $\cos \left(\varphi_{\text {diff }}\right)$ is in the numerator and the denominator, the argument to the $\arctan ()$ formula in Eq. (23) cannot express the absolute sign of the numerator and denominator separately, so an $\arctan ()$ algorithm that gives values from $-\pi / 2$ to $\pi / 2$ should be used. Thus, the wrapped phase term from this formula should have discontinuities of height $h_{d}=\pi$ instead of $2 \pi$. If the other $\arctan ()$ algorithm that gives values from $-\pi$ to $\pi$ is used, then the wrapped phase term is incorrect. After unwrapping, with the full range of $\varphi_{\text {sum }}$ from wavefront shearing in the $x$ direction and Eq. (16a), the full-field $x$ derivative of $\sigma_{1}+\sigma_{2}$ may be determined by

$$
C h \frac{\partial\left(\sigma_{1}+\sigma_{2}\right)}{\partial x}= \pm \frac{p}{2 \pi \tilde{\Delta}} \varphi_{\text {sum }}
$$

\section{Polarization Optics}

Polarization optics such as a linear polarizer, $\lambda / 2$ plate, and $\lambda / 4$ plate allow for manipulation of the input electric field. A general schematic of configurations useful here is shown in Fig. 2. To obtain pure

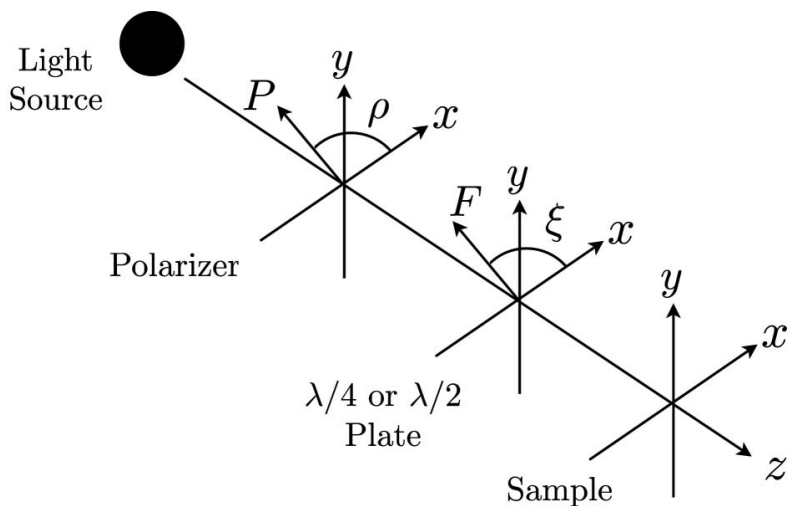

Fig. 2. Polarization optics before the transparent sample: two configurations with either a $\lambda / 4$ or $\lambda / 2$ plate before the sample. 
$E_{x} \hat{1}$ or $E_{y} \otimes$ fields with only a simple change required to switch between the two inputs, a polarizer and a $\lambda / 2$ plate are used; this combination of optics also gives the same range of intensity for both input types, allowing for optimization of the intensity for the experimental equipment, helping to prevent camera saturation. The objective is to start with either pure $E_{x} \hat{1}$ or $E_{y} \otimes$ after the polarizer at $\rho=m \pi / 2, m$ integer, then maintain that field through the $\lambda / 2$ plate with $\xi=\rho$ for the first image, and then obtain the opposite field by setting the $\lambda / 2$ to $\xi=\rho \pm(2 n+1) \pi / 4, n$ integer.

To create circularly polarized light, the collimated laser beam passes through a polarizer with polarization axis at angle $\rho$ to the $x$ axis and then through a $\lambda / 4$ plate with fast axis at angle $\xi$ to the $x$ axis with $\rho-\xi= \pm \pi / 4$. Other combinations of optics can produce the desired equal amplitudes of the $E_{x}$ and $E_{y}$ fields, but for clarity and simplicity, these two configurations are considered here. Table 1 gives the specific polarization optic configurations used in this study, stating the angles of the optics, the amplitudes of the electric field components, and the resultant phase term of the interference pattern in Eq. (15).

\section{Experimental Validation}

The experimental validation was performed on a $12.7 \mathrm{~mm} \times 12.7 \mathrm{~mm}$ square plate with thickness $h=$ $1.0 \mathrm{~mm}$ and with a $60^{\circ} \mathrm{V}$-notch cut out of the side of the plate, as shown in Fig. 3. The depth of the Vnotch, $d$, is $6.35 \mathrm{~mm}$, and the $\overline{\mathrm{V}}$-notch opening width, $w$, is $7.34 \mathrm{~mm}$. The plate is polycarbonate, which is a thermoplastic polymer that is highly photoelastic, with absolute photoelastic constants $A=-2.45 \times$ $10^{-11} \mathrm{~m}^{2} / \mathrm{N}$ and $B=-9.38 \times 10^{-11} \mathrm{~m}^{2} / \mathrm{N}$ [9]. This plastic has a Young's modulus of $E=\overline{2} .3 \mathrm{GPa}$, Poisson ratio of $\nu=0.36$, and refractive index of $n_{o}=1.586$. The specimen is from a polycarbonate sheet with residual stress due to forming, determined to be $\sigma_{11}^{\text {resid }} \approx 1.59 \mathrm{MPa}, \sigma_{22}^{\text {resid }} \approx-1.9 \mathrm{MPa}$, and $\sigma_{12}^{\text {resid }} \approx-0.1 \mathrm{MPa}$. This residual stress is assumed to be constant throughout the material.

In the following example, the sample is compressed by $14.5 \mathrm{~N}(1.14 \mathrm{MPa})$ along the $y$ axis. The experimental optical parameters are the following: the monochromatic CCD camera is an IMPERX IPX-1M48-L with a $1000 \times 1000$ pixel chip; the field of view is $3.77 \mathrm{~mm} \times 3.77 \mathrm{~mm}$; the image resolution is $3.8 \mu \mathrm{m}$; the Ronchi grating pitch, $p$, is $1 \mathrm{~mm} / 40$; the grating separation, $\Delta$, is $12.48 \mathrm{~mm}$; the wavelength of light from the linear polarized $\mathrm{He}-\mathrm{Ne}$ laser is $632.8 \mathrm{~nm}$; and the lateral shearing distance, $d_{\text {shear }}$,

Table 1. Polarization Optic Configurations Used in This Study

\begin{tabular}{cccccc}
\hline $\begin{array}{c}\rho \text { of } \\
\text { Polarizer }\end{array}$ & $\begin{array}{c}\xi \text { of } \lambda / 4 \\
\text { Plate }\end{array}$ & $\begin{array}{c}\xi \text { of } \lambda / 2 \\
\text { Plate }\end{array}$ & $\left|E_{x}\right|$ & $\left|E_{y}\right|$ & $\begin{array}{c}\text { Phase } \\
\text { Determined }\end{array}$ \\
\hline 0 & $\pi / 4$ & - & $A_{x} / \sqrt{2}$ & $A_{x} / \sqrt{2}$ & $\varphi_{\text {sum }}$ \\
0 & - & 0 & $A_{x}$ & 0 & $\varphi_{\text {sum }}+\varphi_{\alpha d}$ \\
0 & - & $\pi / 4$ & 0 & $A_{x}$ & $\varphi_{\text {sum }}-\varphi_{\alpha d}$ \\
\hline
\end{tabular}

is $313 \mu \mathrm{m}$. Williams [15] presented a derivation of the stress fields of a thin plate with an "angular corner" cut out of it under uniaxial tensile load with various boundary conditions. Here, the derivation is applied to a thin plate with a $60^{\circ} \mathrm{V}$-shaped notch under uniaxial compression and is combined with the measured residual stress to obtain the theoretical stress fields and theoretical $\alpha$.

Figure 4 shows the experimental and theoretical images of $\bar{I}_{1}$ for horizontal shear of the configuration shown in Fig. 3. In Figs. 4(a) and 4(c), the images for the pure $E_{x} \hat{i}$ and pure $E_{y} \otimes$ fields, respectively, have interference fringes with good fringe contrast because $I_{c}^{E x}$ and $I_{c}^{E y}$ vary little in the field of view. The image in Fig. $4(\mathrm{e})$ of the $\left|E_{x}\right|=\left|E_{y}\right|$ fields using the $\lambda / 4$ plate method shows discontinuous fringes, evidence of $I_{c}^{\text {circ }}=I_{o}^{\text {circ }} \cos \left(\varphi_{\text {diff }}\right)$ modulating $\cos \left(\varphi_{\text {sum }}\right)$. Clearly, these interference patterns cannot yield the desired phase terms as they are but require phase shifting. Figures $4(\mathrm{~b}), 4(\mathrm{~d})$, and $4(\mathrm{f})$ are the theoretical images for the pure $\overline{E_{x} \hat{1}}$, pure $\overline{E_{y} \bigotimes}$, and $\left|E_{x}\right|=$ $\left|E_{y}\right|$ input fields, which compare well to the experimental fields in shape and fringe density. The slight differences in shape for $|\theta|>\pi / 2$ in these images are most likely due to slight differences in the theoretical and experimental $\alpha$, which is affected by residual stress. The slightly larger lobes near $\theta=0$ are mostly likely due to slightly higher applied stress on this side because of nonuniform compressive loading. Despite these slight differences due to experimental error and residual stress in the material, near $\theta=0$, the experimental image from the pure $E_{x} \hat{1}$ input has the expected wider lobe, the experimental image from the $E_{y} \otimes$ input has the expected narrower lobe, and the experimental image from the $\left|E_{x}\right|=\left|E_{y}\right|$ input field indicates the same interference beading as the theoretical image.

Figure 5 includes the experimental and theoretical wrapped phase fields for $\varphi^{E x}$ and $\varphi^{E y}$. The general three-lobed shape in each experimental field compares well with the theoretical fields, though the differences between the theoretical and experimental are most likely due to slightly nonuniform compressive loading of the sample. The experimental and theoretical wrapped phase field for the $\varphi_{\text {sum }}$ from

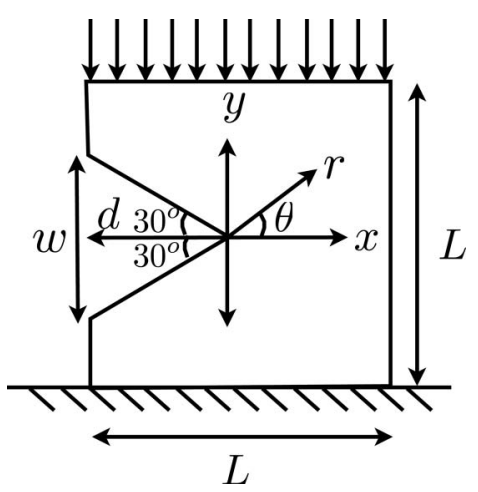

Fig. 3. Schematic of a compressed polycarbonate plate with a side V-notch. 


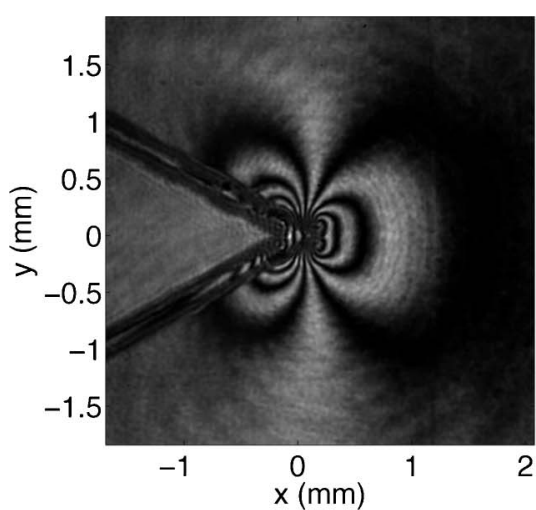

(a)

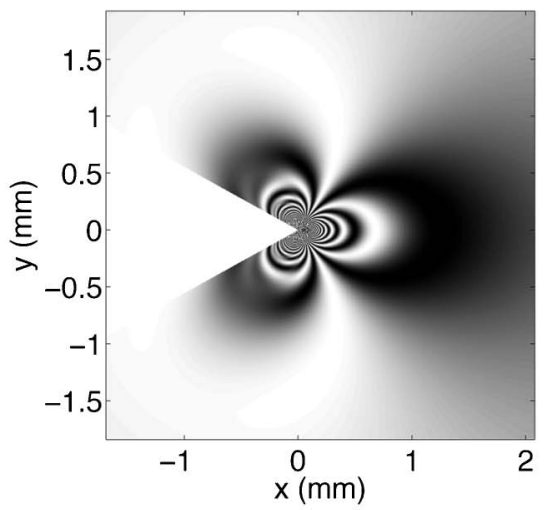

(d)

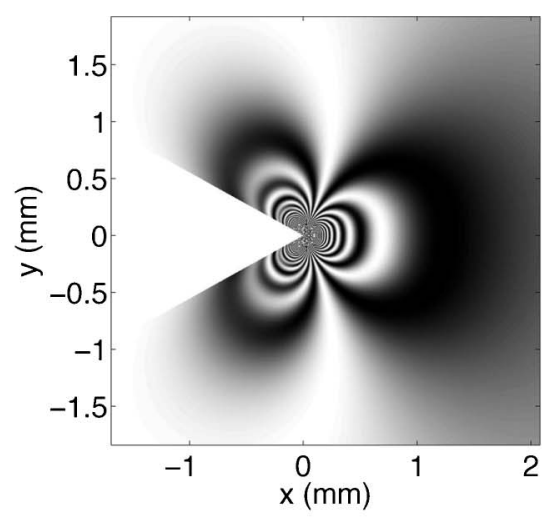

(b)

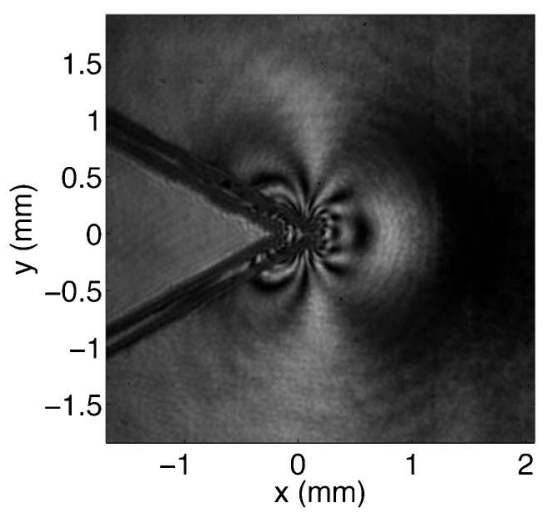

(e)

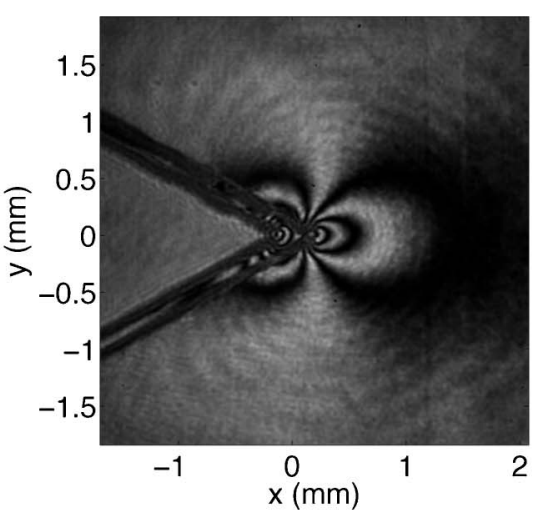

(c)

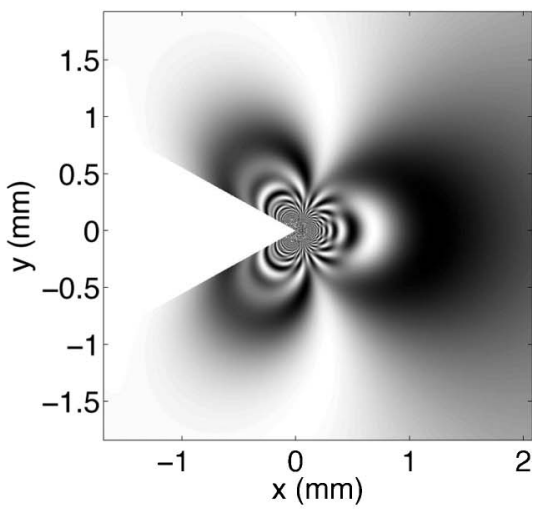

(f)

Fig. 4. Experimental and theoretical images for horizontal shear with good comparison: (a) experimental $I^{E x}=I_{o}^{E x}+I_{c}^{E x} \cos \left[\varphi_{\text {sum }}+\varphi_{\alpha d}\right]$, (b) theoretical $I^{E x}=I_{o}^{E x}+I_{c}^{E x} \cos \left[\varphi_{\text {sum }}+\varphi_{\alpha d}\right]$, (c) experimental $I^{E y}=I_{o}^{E y}+I_{c}^{E y} \cos \left[\varphi_{\text {sum }}-\varphi_{\alpha d}\right]$, (d) theoretical $I^{E y}=I_{o}^{E y}+I_{c}^{E y} \cos \left[\varphi_{\text {sum }}-\varphi_{\alpha d}\right]$, (e) experimental $I^{\text {circ }}=I_{o}^{\text {circ }}+I_{o}^{\text {circ }} \cos \left[\varphi_{\text {diff }}\right] \cos \left[\varphi_{\text {sum }}\right]$, and (f) theoretical $I^{\text {circ }}=I_{o}^{\text {circ }}+I_{o}^{\text {circ }} \cos \left[\varphi_{\text {diff }}\right] \cos \left[\varphi_{\text {sum }}\right]$ (note: the V-notch region is masked in white in the theoretical images).

Eq. (23) from the $\lambda / 4$ plate method and the theoretical $\cos \left(\varphi_{\text {diff }}\right)$ field are shown in Fig. 6. In Fig. 6(a), the fringes have regions in a four-lobed clover leaf pattern with greater noise and scatter, which corresponds to regions near $\cos \left(\varphi_{\text {diff }}\right)=0$ boundaries found in Fig. 6(c), which is expected since Eq. (23) is indeterminate for $\cos \left(\varphi_{\text {diff }}\right)=0$. The theoretical wrapped $\varphi_{\text {sum }}$ field in Fig. 6(b) does not have these poor contrast regions because the synthetic data have exact cancellation of the $\cos \left(\varphi_{\text {diff }}\right)$ in the $\arctan ()$ formula.

The wrapped phase terms are unwrapped using a weighted preconditioned conjugate gradient method, a robust two-dimensional phase unwrapping method for interferometric fringes with noise developed by Ghiglia and Romero [16]. To reduce unwrapping errors, a weight function considers the reliability of the wrapped phase information from experimental

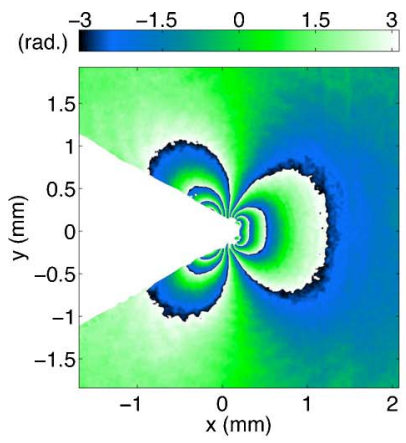

(a)

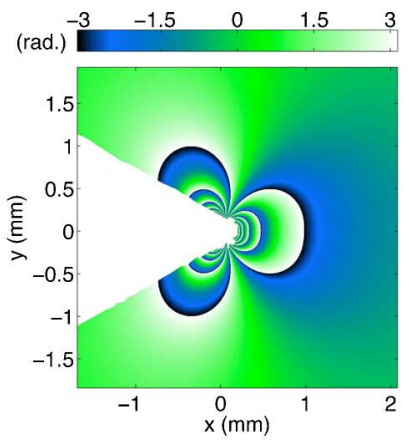

(b)

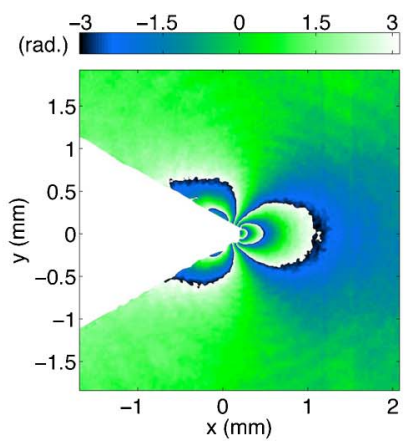

(c)

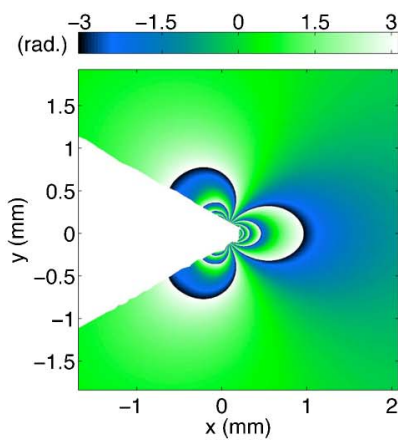

(d)

Fig. 5. (Color online) Experimental and theoretical wrapped phase maps (in radians) with the V-notch masked in white: (a) experimental $\varphi^{E x}=\varphi_{\text {sum }}+\varphi_{\alpha d}$, (b) theoretical $\varphi^{E x}=\varphi_{\text {sum }}+\varphi_{\alpha d},(\mathrm{c})$ experimental $\varphi^{E y}=\varphi_{\text {sum }}-\varphi_{\alpha d}$, and (d) theoretical $\varphi^{E y}=\varphi_{\text {sum }}-\varphi_{\alpha d}$. 


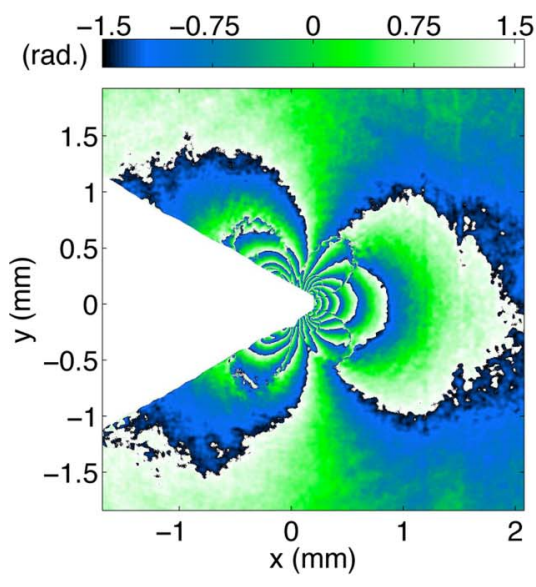

(a)

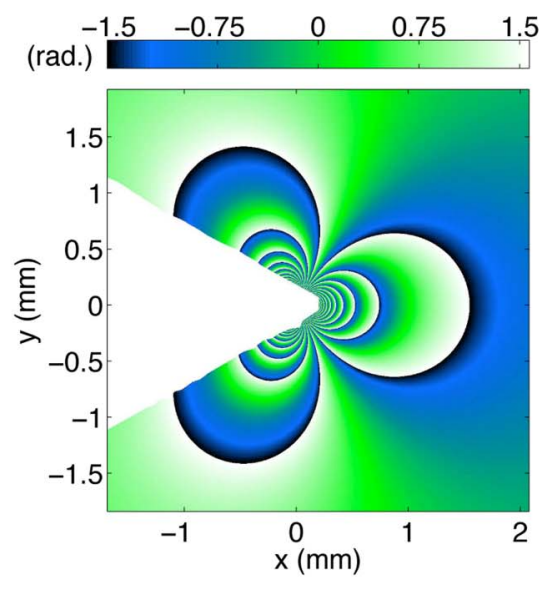

(b)

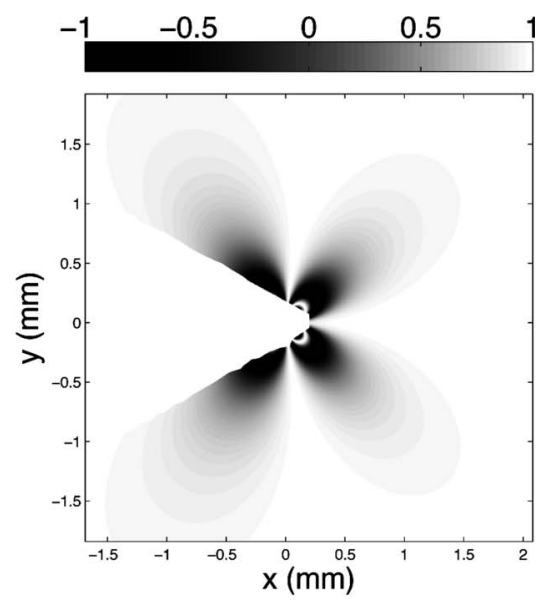

(c)

Fig. 6. (Color online) Wrapped phase maps from $\lambda / 4$ plate method (in radians) with the V-notch masked in white: (a) experimental $\varphi_{\text {sum }}$ for $\cos \left(\varphi_{\text {diff }}\right) \neq 0$, (b) theoretical $\varphi_{\text {sum }}$, and (c) theoretical $\cos \left(\varphi_{\text {diff }}\right)$ field with its four-lobed clover leaf pattern.

data and physical boundaries. The reliability condition considers pixelwise differences in phase in the wrapped phase term; differences of size $h$ or nearly zero receive a weight of close to one since the wrapped phase term is expected to be either continuous or have a jump of $h$, while differences of $h / 2$ are considered unreliable and given a weight of zero. The following formula is applied to each pixel to develop the weight function $\mathbf{W}$, where $\Delta \psi_{k}$ is the wrapped phase difference between the $k$ th nearest neighbor of the $(i, j)$ pixel [17]:

$$
W_{i, \bigotimes}=\prod_{k=1}^{8} \frac{1}{2}\left\{\cos \left(2 \pi \frac{\Delta \psi_{k}}{h}\right)+1\right\} .
$$

Additionally, physical boundaries and regions in the field with no photoelastic material, as with the Vnotch in the example, are given a weight of zero. Based on a priori knowledge of the experiment, the weight of regions with high concentrations of fringes that cannot be resolved with the given pixel resolution are also set to zero to reduce unwrapping errors near these regions.

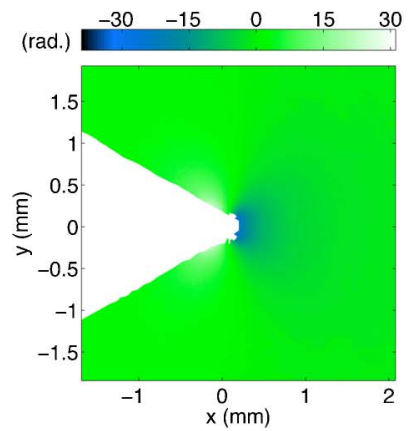

(a)

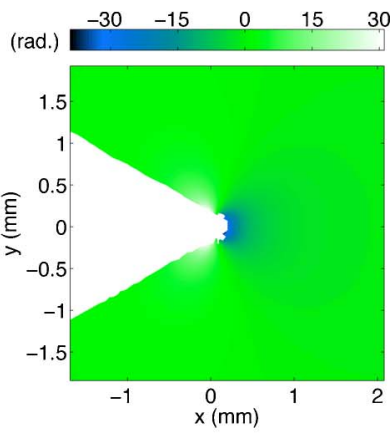

(b)

Figure 7 shows the unwrapped $\varphi^{E x}$ and $\varphi^{E y}$ fields for experimental and theoretical data. The preconditioned conjugate gradient method successfully unwraps the phase discontinuities in these fields; the data from the air in the V-notch region do not propagate into the polycarbonate data due to the weight function; unwrapped $\varphi^{E x}$ and $\varphi^{E y}$, like the theoretical fields, have a general monotonic increase or decrease as $r \rightarrow 0$ toward the notch tip.

Figure 8(a) is the experimental $\varphi_{\text {sum }}$ determined by $\left(\varphi^{E x}+\varphi^{E \bar{y}}\right) / 2$, and Fig. 8(b) is the unwrapped experimental $\varphi_{\text {sum }}$ from the $\lambda / 4$ plate method. In comparison, qualitatively, the $\varphi_{\text {sum }}$ field from the $\lambda / 4$ plate method does not agree with the theoretical field in Fig. 8(c) as well as $\varphi_{\text {sum }}$ from the pure $E_{x} \hat{1}$ and pure $E_{y} \otimes$ fields agrees with the theoretical field; some minor unwrapping errors are evident in Fig. 8(b) near the $\cos \left(\varphi_{\text {diff }}\right)=0$ regions in the four-lobed clover leaf pattern seen in Fig. 6(c). Additionally, the experimental $\varphi_{\alpha d}$ in Fig. 8(d) from the $\left(\varphi^{E x}-\varphi^{E y}\right) / 2$ has a four-lobed clover leaf pattern like the theoretical $\varphi_{\alpha d}$ field in Fig. 8(e).

One measure of the global error is the root mean square deviation (RMSD) normalized by the range

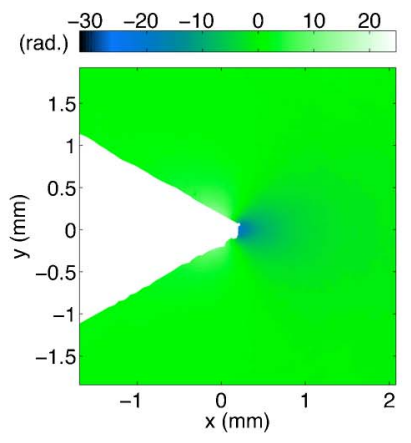

(c)

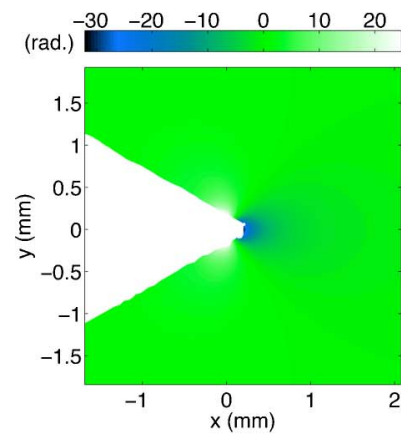

(d)

Fig. 7. (Color online) Experimental and theoretical unwrapped phase term from the pure $E_{x} \hat{i}$ and pure $E_{y} \otimes$ fields (in radians) with the Vnotch masked in white: (a) experimental $\varphi^{E x}$, (b) theoretical $\varphi^{E x}$, (c) experimental $\varphi^{E y}$, and (d) theoretical $\varphi^{E y}$. 


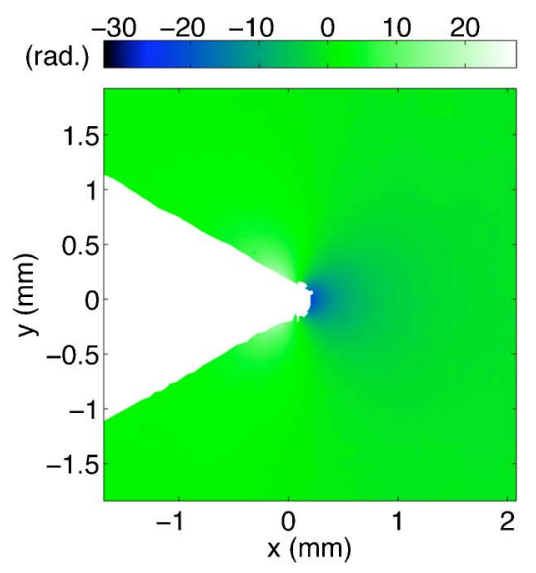

(a)

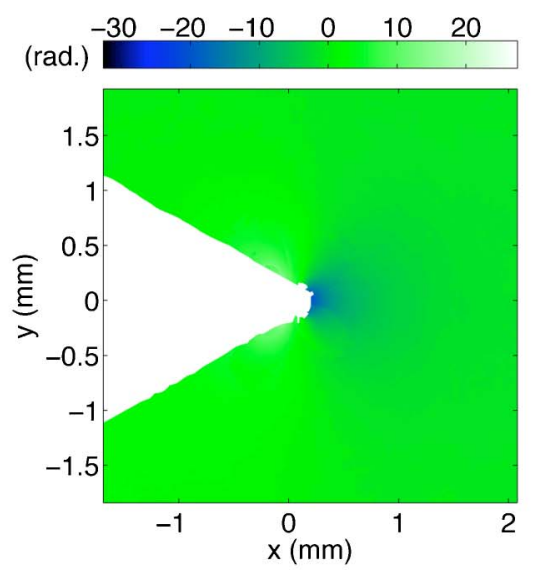

(b)

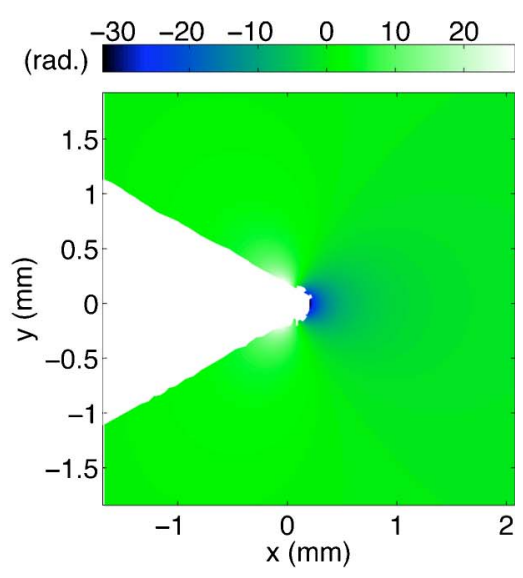

(c)

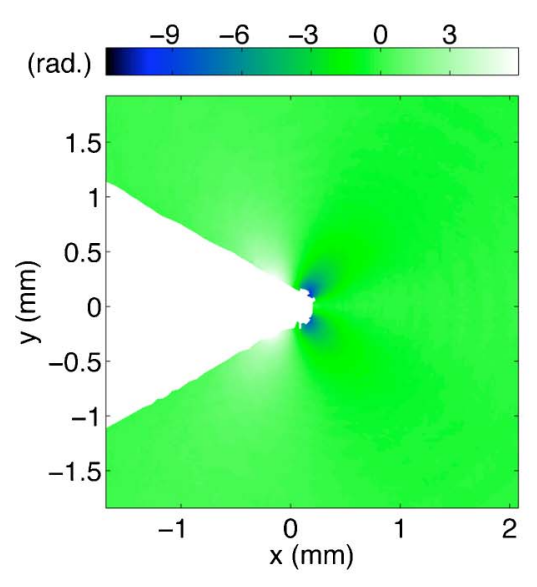

(e)

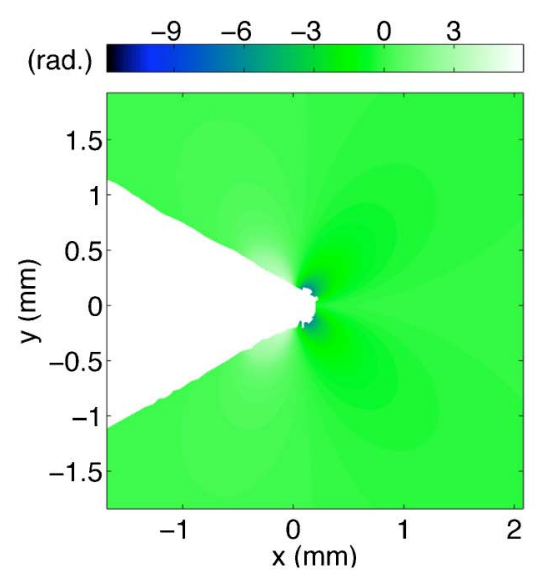

(f)

Fig. 8. (Color online) Experimental and theoretical phase maps of $\varphi_{\text {sum }}$ and $\varphi_{\text {diff }}$ (in radians) with the V-notch masked in white: (a) experimental $\varphi_{\text {sum }}=\left(\varphi^{E x}+\varphi^{E y}\right) / 2$, (b) experimental $\varphi_{\text {sum }}$ from the $\lambda / 4$ method, (c) theoretical $\varphi_{\text {sum }}$, (d) experimental $\varphi_{\alpha d}=\left(\varphi^{E x}-\varphi^{E y}\right) / 2$, and (e) theoretical $\varphi_{\alpha d}$.

of experimental data, denoted NRMSD. Only data points not masked by the notch mask are considered here. Table 2 reports the error analysis of several fields. The NRMSD is low for each of the fields, with the largest error in the $\varphi^{E y}$ at only $2.1 \%$. As is evident in Figs. 9(a) and 9(b), which show the difference between the theoretical and the two experimental $\varphi_{\text {sum }}$ fields, the greatest errors are close to the notch tip, which is understandable since the stress derivative changes so rapidly near the notch tip that the small $d_{\text {shear }}$ assumption, which allows the phase to be related to stress derivatives in Eq. (13), breaks down. The unwrapping errors due to the $\cos \left(\varphi_{\text {sum }}\right)=0$ regions are in the four-lobed clover leaf pattern in Fig. $9(\mathrm{~b})$, leading to a slightly higher NRMSD for the $\overline{\varphi_{\text {sum }}}$ from the $\lambda / 4$ plate method than for the $\varphi_{\text {sum }}$ from the pure $E_{x} \hat{1}$ and pure $E_{y} \otimes$ fields data. Both methods of determining $\varphi_{\text {sum }}$ give reasonable global error, though the pure $E_{x} \hat{1}$ and pure $E_{y} \otimes$ fields method does seem to better confine the error near the notch tip and is not affected the $\cos \left(\varphi_{\text {diff }}\right)$ issue. Another benefit of the pure $E_{x} \hat{1}$ and pure $E_{y} \otimes$ fields method is the determination of $\varphi_{\alpha d}$, which has low error as well; the difference between the theoretical and experimental $\varphi_{\alpha d}$ is shown in Fig. 9(c), confining the error to near the notch tip. The excellent agreement of the experimental data with theoretical data in this example demonstrates that the use of polarization optics and phase shifting can successfully extract phase data from complicated interference images that have physical meaning in terms of stress in the photoelastic material, as explained in the previous analysis in Subsection 2.B.

Table 2. Error Analysis for Various Experimental Fields for Horizontal Shear

\begin{tabular}{lccc}
\hline \multicolumn{1}{c}{ Phase } & $\begin{array}{c}\text { RMSD } \\
(\text { Rad })\end{array}$ & $\begin{array}{c}\text { Data } \\
\text { Range (Rad) }\end{array}$ & $\begin{array}{c}\text { NRMSD } \\
\text { (No Units) }\end{array}$ \\
\hline$\varphi^{E x}$ & 0.73 & 49.14 & 0.015 \\
$\varphi^{E y}$ & 0.52 & 34.05 & 0.015 \\
$\varphi_{\text {sum }}$ from $\lambda / 4$ method & 0.72 & 34.57 & 0.021 \\
$\varphi_{\text {sum }}=\left(\varphi^{E x}+\varphi^{E y}\right) / 2$ method & 0.57 & 38.94 & 0.015 \\
$\varphi_{\alpha d}=\left(\varphi^{E x}-\varphi^{E y}\right) / 2$ method & 0.26 & 17.85 & 0.015 \\
\hline
\end{tabular}




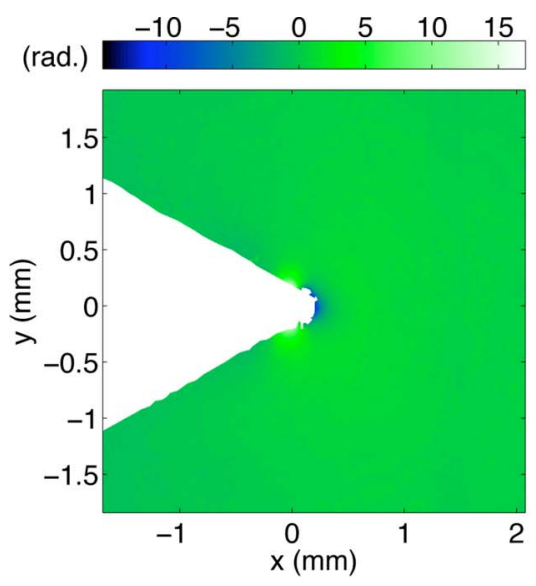

(a)

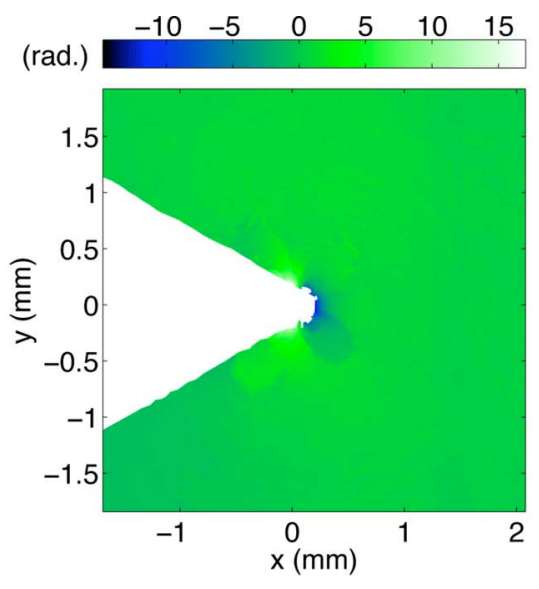

(b)

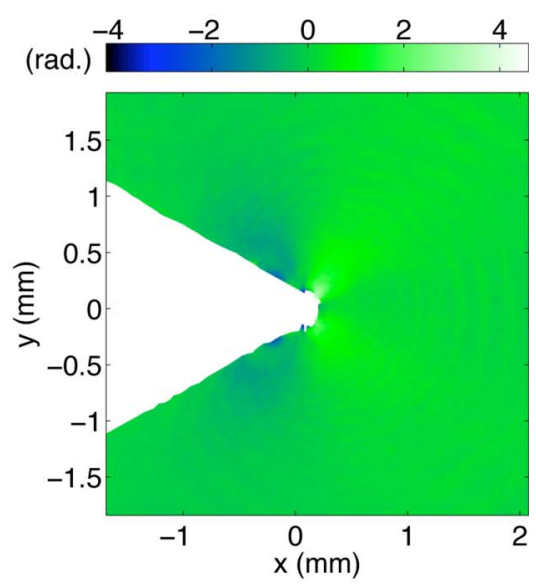

(c)

Fig. 9. (Color online) Difference between theoretical and experimental $\varphi_{\text {sum }}$ and $\varphi_{\alpha d}$ (in radians) with the V-notch masked in white: (a) comparison for $\varphi_{\text {sum }}=\left(\varphi^{E x}+\varphi^{E y}\right) / 2$, (b) comparison for $\varphi_{\text {sum }}$ from the $\lambda / 4$ method, and (c) comparison for $\varphi_{\alpha d}=\left(\varphi^{E x}-\varphi^{E y}\right) / 2$.

\section{Conclusions}

Wavefront shearing interferometry, specifically coherent gradient sensing (CGS), is used to analyze a wavefront transmitted through a photoelastic material. A detailed analysis of the transmitted wavefront properties, of the lateral shearing, and of the resulting interference patterns is provided for a general wavefront shearing interferometer, with some specialization for CGS. Phase information related to stress gradients in a deformed photoelastic material may be extracted from the complicated interference pattern by the use of polarization optics and phase shifting. This is experimentally validated using CGS on a compressed polycarbonate plate with a V-notch. Using this general analysis, stress information may be obtained in full-field for photoelastic materials with input electric field polarization control and any phase shifting transmission wavefront shearing interferometry.

We gratefully acknowledge the support of the National Science Foundation (NSF) (DMR \# 0520565) through the Center for Science and Engineering of Materials (CSEM) at the California Institute of Technology, of the American Society for Engineering Education National Defense Science and Engineering Graduate (NDSEG) Fellowship Program, and of the National Science Foundation Graduate Research Fellowship Program. We thank Michael Mello for his insights and help during this project.

\section{Reference}

1. M. Murty, "The use of a single plane parallel plate as a lateral shearing interferometer with a visible gas laser source," Appl. Opt. 3, 531-534 (1964).

2. T. Park, S. Suresh, A. Rosakis, and J. Ryu, "Measurement of full-field curvatue and geometrical instability of thin filmsubstrate systems through cgs interferometry,” J. Mech. Phys. Solids 51, 2191-2211 (2003).

3. H. Tippur, S. Krishnaswamy, and A. Rosakis, "A coherent gradient sensor for crack tip deformation measurements: analysis and experimental results," Int. J. Fracture 48, 193-204 (1991).
4. H. Tippur, S. Krishnaswamy, and A. Rosakis, "Optical mapping of crack tip deformations using the methods of trasmission and reflection coherent gradient sensing: a study of crack tip $k$-dominance," Int. J. Fracture 52, 91-117 (1991).

5. A. J. Rosakis, "Optical techniques sensitve to gradients of optical path difference: the method of caustics and the coherent gradient sensor (CGS)," in Experimental Techniques in Fracture, J. S. Epstein, ed. (Wiley, 1993), pp. 327-425.

6. S. Krishnaswamy, "Photomechanics," in Techniques for NonBirefringnet Objects: Coherent Shearing Interferometry and Caustics, Vol. 77 of Topics in Applied Physics (SpringerVerlag, 2000), pp. 295-321.

7. G. A. Papadopoulos, Fracture Mechanics: the Experimental Method of Caustics and the Det. Criterion of Fracture (Springer-Verlag, 1993).

8. A. Kobayashi, ed., Handbook of Experimental Mechanics (Wiley, 1993).

9. K. Shimizu, M. Suetsugu, T. Nakamura, and S. Takahashi, "Evaluation of concentrated load by caustics and its application in the measurement of optical constant," JSME Int. J. 41, 134-141 (1998).

10. H. Lee, A. Rosakis, and L. Freund, "Full-field optical measurement of curvatures in unltra-thin-film-substrate systems in the range of geometrically nonlinear deformations," J. Appl. Phys. 89, 6116-6129 (2001).

11. C. Liu, A. Rosakis, R. Ellis, and M. Stout, "A study of the fracture behavior of unidirectional fiber-reinforced composites using coherent gradient sensing (CGS) interferometry," Int. J. Fracture 90, 355-382 (1998).

12. E. Coker and L. Filon, A Treatise on Photo-elasticity (Cambridge U. Press, 1993).

13. M. Frocht, Photoelasticity (Wiley, 1941), Vol. 1.

14. T. Narasimhamurty, Photoelastic and Electro-optic Properties of Crystals (Plenum, 1981).

15. M. Williams, "Stress singularities resulting from various boundary conditions in angular corners of plates in extension," J. Appl. Mech. 19, 526-528 (1952).

16. D. Ghiglia and L. Romero, "Robust two-dimensional weighted and unweighted phase unwrapping that uses fast transforms and iterative methods," J. Opt. Soc. Am. A 11, 107-117 (1994).

17. A. Baldi, F. Bertolino, and F. Ginesu, "On the performance of some unwrapping algorithms," Opt. Laser Eng. 37, 313-330 (2002). 